\title{
09 ECOLOGY AND EPIDEMIOLOGY
}

09.001 - URBAN BLASTOMYCOSIS: A TALE OF TWO CITIES

Baumgardner, D. J. ${ }^{1}$; Knavel, E. M. ${ }^{2}$; Steber, D. ${ }^{3}$; Swain, G. R. ${ }^{4}$

${ }^{1,2}$ University of Wisconsin Medical School - Family Medicine; ${ }^{3}$ Center for Urban Population Health - Aurora Health Care; ${ }^{4}$ City of Milwaukee - Health Department

Introduction: Most studies of endemic and outbreak blastomycosis have involved rural areas, and case homesites have been associated with waterways and sand soils. Objectives: To compare environmental and homesite features associated with blastomycosis in the cities of Eagle River (ER pop. 2000) [ $n=25$ ] and Milwaukee County (MK pop. 940,000) [n=45], Wisconsin. Methods: Comparison of homesites of mandatory State-reported cases of human blastomycosis. Controls for ER were 57 random number selected geographically overlapping households; and four nearest same-street addresses were controls for ER and MK. Power analysis guided number of controls. ARC-GIS was used to geocode addresses and to observe geographic features of homesites. Cluster analysis was performed with CrimeStatII. Each case and control property was directly observed. Categorical data was analyzed using Yates corrected chi-square or Fisher's exact test; continuous variables by Mann-Whitney test. Results: Cases averaged $354 \mathrm{~m}$ to waterways in ER and $1067 \mathrm{~m}$ in MK. No MK homesites were on sand soils compared to $24 / 25$ for ER. One case cluster was seen on Milwaukee's North side ( 2 zip codes) where the estimated annual incidence was 2.8/100,000 compared to $0.96 / 100,000$ for all MK County (versus 74/100,000 for ER). In both cities, homesite features of lot size/type, approximate home age, garage, driveway, foundation, and forestation did not differ from controls. In ER, porches were more common among case houses (10/17 versus $16 / 65$ same-street controls [ $\mathrm{p}=0.02]$ versus $15 / 51$ random controls $[\mathrm{p}=0.06])$. In MK, cases were less common in the most urbanized watersheds $(0.49 / 100,000 / \mathrm{yr})$ vs. Lake Michigan shores $(0.85)$ vs. remaining three open watersheds (1.4) $[\mathrm{p}=0.01]$. Conclusion: Blastomycosis incidence rates and geographic associations differ between these two Wisconsin cities separated by $323 \mathrm{~km}$. Despite low incidence rates, blastomycosis cases still appear to vary geographically and by associated waterway features in a large urban area. Financial support: Donation to St. Luke's Foundation by Mr. and Mrs. Charles Goldsworthy, Eagle River, WI; UW Department of Family Medicine

\subsection{2 - PARACOCCIDIOIDOMYCOSIS-INFECTION IN FREE-LIVING} MONKEYS OF NORTHWEST PARANÁ STATE, BRAZIL

Corte, A. C. ${ }^{1}$; Itano, E. N. ${ }^{1}$; Ono, M. A. ${ }^{1}$; Malanski, L. S. ${ }^{2}$; Navarro, I. T. ${ }^{2}$; Shiozawa, M. M. ${ }^{2}$; Svoboda, W. K. ${ }^{2}$; Aguiar, L. M. ${ }^{3}$; Passos, F. C. ${ }^{3}$; Camargo, Z. P. ${ }^{4}$

${ }^{1}$ Universidade Estadual de Londrina - Departamento de Ciências Patológicas; ${ }^{1,1}$ Universidade Estadual de Londrina - Ciências Patológicas; ${ }^{2,2,2}$ Universidade Estadual de Londrina Departamento de Medicina Veterinária Preventiva; ${ }^{2}$ Universidade Estadual de Londrina Departamento de Medicina Veterinária Preventiva; ${ }^{3}$ Universidade Federal do Paraná Departamento de Zoologia; ${ }^{3}$ Universiade Federal do Paraná - Departamento de Zoologia; ${ }^{4}$ Universidade Federal de São Paulo - Microbiologia e Imunologia e Parasitologia

Paracoccidioidomycosis (PCM) is a deep mycosis with chronic evolution and granulomatous pattern caused by the dimorphic fungus Paracoccidioides brasiliensis. It is the most prevalent systemic mycosis in Latin América, especially in Brazil, with the highest number of cases. The patients are mainly rural workers, between 30-60 years old. Little is known about the ecology of $P$. brasiliensis, although much progress has been achieved in studies on the pathogenesis and diagnosis of PCM. The aim of this work was evaluate $P$. brasiliensis infection in $C$. apella monkeys. A total of 39 serum samples were collected from monkeys captureded in the region of Porto Rico, Paraná. The samples were analyzed by ELISA, using gp43 as antigen and protein-A-peroxidase as conjugate. The seropositivity was $64,1 \%$. This result suggests that $C$. apella monkeys play a role in the $P$. brasiliensis ecology. Financial support: CNPq, Fundação Araucária, Fundo Paraná Tecnologia, CAPES

\subsection{3 - PARACOCCIDIOIDOMYCOSIS-INFECTION IN HORSES IN THE} NORTH PARANÁ STATE, BRAZIL

\section{Corte, A. C. ${ }^{1}$; Itano, E. N. ${ }^{1}$; Ono, M. A. ${ }^{1}$; Camargo, Z. P. ${ }^{2}$}

${ }^{1,1}$ Universidade Estadual de Londrina - Ciências Patológicas, ${ }^{1}$ Universidade Estadual de Londrina - Departamento de Ciências Patológicas; ${ }^{2}$ Universidade Federal de São Paulo - Microbiologia e Imunologia e Parasitologia

The paracoccidiodoycosis (PCM) is the most prevalent systemic mycosis in Latin América, specially in Brazil, that shows the higher number of cases. It is caused by the dimorphic fungus Paracoccidioides brasiliensis, that grows in the filament form at room temperature and in yeast form at $37^{\circ} \mathrm{C}$ or in the host. The PCM can be classified in PCMinfection and PCM-disease. The first one occurs in individuals of both sexes, healthy, that live or lived in endemic areas and not developed the disease; the latter affect mainly male rural workers, between 30-60 years old. Besides the armadillos, the participation of other animal species in the eco-epidemiology of PCM is unclear. The aim of this work was to evaluate the infection of horses by $P$. brasiliensis. The serum samples were collected from 100 horses of Northern Region of Paraná State and analyzed by ELISA using gp43 as antigen and protein-G-peroxidase as conjugate. The animals showed positivity of $22 \%$. This result indicates that horses also can be infected by P. brasiliensis and therefore may be used as a PCM epidemiological marker. Financial support: CNPq, CAPES, Fundação Araucária.

\subsection{4 - ATTEMPTS TO ISOLATE PARACOCCIDIOIDES BRASILIENSIS FROM BATS CAPTURED IN MINAS GERAIS STATE OF BRAZIL}

Silva-Vergara, M. L. ${ }^{1}$; Bento, E. C. ${ }^{2}$; Costa, D. F. ${ }^{3}$; Costa, T. F. ${ }^{4}$; Santos, C. T. B. ${ }^{5}$; Ramirez, L. E. ${ }^{6}$

${ }^{1}$ Universidade Federal do Triângulo Mineiro - Departamento de Clínica Médica; ${ }^{2}$ Universidade Federal do Triângulo Mineiro - Curso de Pós-Graduação em Medicina Tropical e Infectologia da U.F.T.M, Departamento de Clínica Médica; ${ }^{3,4}$ Universidade Federal do Triângulo Mineiro Acadêmico de Medicina, Departamento de Clínica Médica; ${ }^{5}$ Universidade Federal do Triângulo Mineiro - Laboratório de Micologia, Departamento de Clínica Médica; ${ }^{6}$ Universidade Federal do Triângulo Mineiro - Disciplina de Parasitologia, Departamento de Ciências Biológicas

Introduction: Chiroptean species can carrier saprophytic and pathogenic fungi mainly Histoplasma capsulatum Public.Health.Report. 73: 590-595, 1958, Criptococcus sp Sabouraudia. 6: 127-132, 1968, Candida sp Appl.Environ.Microbiol 44: 570-575, 1982, coccidioides immitis Life Sci. 22: 679-684, 1984, dermatophyte species Trop.Geogr.Med 18:260-263, 1966, and rarely Paracoccidioides brasiliensis Sabouraudia 4:124-125, 1965, Sabouraudia 19: 165-168, 1981. Ecologic niche from the later hasn't still been elucidated despite the great deal of studies carried out in endemic areas. The purpose of this work was to try to recover Paracoccidioides brasiliensis from bats' viscera. Methods: Previous license from Brazilian institute of environment (IBAMA), 237 bats from fifteen species were captured in Uberaba, Conquista, Perdizes and Água Comprida, cities localized in the Triângulo Mineiro region. Bats received anesthesia with ethyl ether, and were autopsied and had theirs lungs, liver, spleen and heart removed. Small fragments from each organ were inoculated onto Mycosel ${ }^{R}$ and BHI agar (3 tubes for each organ) and the cultures were observed during 12 weeks. Results: Despite the great quantity of cultures performed, no dimorphic fungus similar to $P$. brasiliensis were observed. From liver of four individuals of two frugivorous bats (Carollia perspicillata and Sturnira lilium) were isolated three Candida krusei and one yeastlike unidentified. Other two C. krusei specimens were recovered from liver of two Glossophaga soricina individuals (a nectarivorous bat). One C. krusei and other yeast like unidentified were isolated from spleen of two Carollia perspicillata. Two other individuals of this species also permitted the isolation of one Trichosporum $s p$ and one Scytalidium $s p$. From lung of one Desmodus rotundus (an hematophagous bat) was obtained one Paecilomyces sp. One Trichosporum sp and one yeastlike unidentified were isolated from heart of Carollia perspicillata and Desmodus rotundus, respectively. Discussion: Fourty years ago, $P$. brasiliensis was recovered from digestive tract of the frugivorous bats in Colombia; other attempts to repeat this finding were unsuccessful. Negative results, despite the great number of cultures carried out, confirm the great difficulty to characterize its natural habitat in soi and/or mammals reservoires. The isolation of several pathogenic fungi from different bats' species is according to other report from Brazilian Amazon, and confirm the potential of bats to be a natural source infection. Conclusion: The relationship between bats and $P$. brasiliensis may be an accidental and rare event. Financial support: CAPES/FAPEMIG

\subsection{5 - MORPHOLOGICAL AND MOLECULAR CHARACTERIZATION OF THE FIRST ISOLATE OF PARACOCCIDIOIDES BRASILIENSIS FROM DOG} (CANIS FAMILIARIS)

Bosco, S. M. G. ${ }^{1}$; Theodoro, R. C. ${ }^{2}$; Macoris, S. A. G. ${ }^{3}$; Farias, M. R. ${ }^{4}$; Muro, M. ${ }^{5}$; Ribeiro, M. G. ${ }^{6}$; Bagagli, E. ${ }^{7}$

${ }^{1,2,3,7}$ Instituto de Biociências de Botucatu/UNESP - Microbiologia e Imunologia; ${ }^{4}$ Pontifícia Universidade Católica do Paraná - Clínica Veterinária, ${ }^{5}$ Universidade Federal do Paraná - Hospital das Clínicas; ${ }^{6}$ Faculdade de Medicina Veterinária e Zootecnia/UNESP Botucatu - Higiene Veterinária e Saúde Pública

Introduction and Objectives: Paracoccidioides brasiliensis is the etiological agent of Paracoccidioidomycosis, the most important and prevalent systemic mycosis of Latin America. This fungus has frequently been isolated from human clinical samples and armadillo tissues. The infection in dogs is reported in serological surveys and the disease has been reported recently by histopathological, imunohistochemical and molecular procedures. This work aimed to characterize morphologically and molecularly the first isolate of $P$. brasiliensis obtained recently from a dog. Methods and Results: The morphological characterization consisted of evaluating the dimorphism by culturing the isolate at 25,35 and $37^{\circ} \mathrm{C}$ on GlucosePeptone-Yeast Extract (GPY). Slide culture was carried out with GPY medium at 30, 32, 34 and $36^{\circ} \mathrm{C}$ to determine the transition temperature. For the molecular characterization, DNA was obtained in the yeast phase and the amplicons of gp43 gene and rDNA region (ITS15.8S-ITS2) were submitted to sequencing in both strands in a MegaBace ${ }^{\mathrm{TM}} 1000$ (Amershan Biosciences) and the reactions were performed according to DYEnamic ET Dye Terminator Cycle Sequencing Kit (Amershan Biosciences). The sense and anti-sense sequences were aligned by Clustal W Software and submitted to BLASTn (Basic Local Search Alignmen Tool for nucleotide). The macroscopic aspect of the mycelial phase was typical cotton-like surface with presence of fissures and a diffusible brown pigment in the agar. Microscopically, 
few structures were observed similar to conidia and other chlamydospores. The colony of the yeast phase showed the typical cream-like color but was smooth in topography. Microscopy presented the multiple-budding cells characteristic of this pathogen. The transition temperature was $34^{\circ} \mathrm{C}$ and the growth and viability of the cells were better observed at $37^{\circ} \mathrm{C}$. The molecula analysis showed that this isolate present a similarity of $98 \%$ (score 1063 and e-value 0.0 ) and $99 \%$ (score 1090 and e-value 0.0 ) with P. brasiliensis according to the sequencing of 591 nucleotides from gp 43 gene and 625 nucleotides from ITS-5.8S region, respectively. Conclusion: This first $P$. brasiliensis isolate from a dog was successfully characterized in both microbiological and molecular features. Both infection and disease need to be investigated further in dogs, since this animal specie is closely related to man and may play an important role in the epidemiological chain of PCM. As observed in North American Blastomycosis, dogs are very sensitive to the development of this disease, which has been considered a harbinger for human cases of Blastomycosis. Since there are no pathognomonic symptoms of PCM in dogs, differential diagnosis must be considered. The combined use of molecular tools may improve this diagnosis and facilitate eco-epidemiological studies of this important systemic mycosis. Financial support: FAPESP

\subsection{6 - MOLECULAR DETECTION OF PARACOCCIDIOIDES BRASILIENSIS DNA IN STOOL AND TISSUE SAMPLES OF NATURALLY INFECTED ARMADILLOS}

Bosco, S. M. G. ${ }^{1}$; Rosa, P. S. ${ }^{2}$; Simões, L. B. ${ }^{3}$; Theodoro, R. C. ${ }^{3}$; Macoris, S. A. G. ${ }^{4}$; Pereira Jr., H. R. J. ${ }^{5}$; Franco, M. ${ }^{6}$; Bagagli, E. ${ }^{\text {? }}$

1,3,4,5,7Instituto de Biociências de Botucatu/UNESP - Microbiologia e Imunologia; ${ }^{2}$ Instituto Lauro de Souza Lima, Bauru/SP - Clínica Médica; ${ }^{3}$ Instituto de Biociências de Botucatu/UNESP Departamento de Microbiologia e Imunologia; ${ }^{6}$ UNIFESP - Patologia

Introduction and Objectives: Paracoccidioidomycosis (PCM) is a systemic mycosis caused by Paracoccidioides brasiliensis, a thermo-dimorphic fungus whose ecology remain enigmatic. The finding that the nine-banded armadillo Dasypus novemcinctus is naturally infected by this pathogen opened new opportunities to study its ecology, epidemiology and other aspects of the host/pathogen interactions. Is this relation fortuitous or obligatory? Can the armadillo develop PCM disease? Could the armadillo eliminate the fungus to the environment, contributing to dissemination of the pathogen in nature? Methods and Results: With the authorization of the Brazilian Protection Agency (IBAMA), we captured 8 armadillos ( 3 female and 5 male) in a hyper-endemic area of PCM (Manduri County) and one animal (male) in a fluvial island of Barra Bonita Dam, Tietê River, São Manuel County, São Paulo State, Brazil. The 8 Manduri animals were kept under captive conditions for the developmen of PCM disease at Instituto Lauro de Souza Lima, and were periodically observed. The island animal was sacrificed and evaluated by fragment culture, histopathological and molecular assays using Nested-PCR reactions with rDNA sets of primers. The ecological data from the island were also evaluated using the Geographic Information System (GIS). Four animals died naturally after respective periods of $1,4,8$ and 9 months in captivity and another had to be killed after 26 months of captivity. Culture and histopathological analysis did not show the presence of the fungus in tissues and the molecular assays displayed the DNA of the fungus in the spleen, lung and mesenteric lymph nodes in two animals. Three other animals remain alive in captivity and seem to be in good health. One of them tested positive by the Nested-PCR reaction carried out in stool sample after 9 months of evaluation. Organ cultures from the island armadillo were positive in fragments of spleen and mesenteric lymph nodes. Histopathology was negative and PCR showed a specific fragment of $P$ brasiliensis DNA in stool sample. Mean annual precipitation and temperature of the island are, respectively, $1383 \mathrm{~mm}$ and $22.07^{\circ} \mathrm{C}$, whereas the altitude is slightly less than 450 m.Conclusion: These data seem to indicate that armadillos can harbor the fungus without active disease or perhaps longer periods are necessary to develop PCM. The detection of specific DNA of $P$. brasiliensis in armadillo stools may suggest a possible gastroenteric cycle of this pathogen in this animal species. The detection of an isolated population of naturally infected armadillos represents a new and promising strategy to pinpoint the whereabouts of P. brasiliensis in nature. Financial support: FAPESP (grants 01/13026-0, 02/00466-5 and 02/04489-0)

\subsection{7 - ECOLOGICAL STUDY OF PARACOCCIDIOIDES BRASILIENSIS IN} SOIL: CULTURE AND MOLECULAR DETECTION

Terçarioli, G. R. ${ }^{1}$; Theodoro, R. C. ${ }^{2}$; Bosco, S. M. G. ${ }^{3}$; Reis, G. M. ${ }^{4}$; Macoris, S. A. G. ${ }^{5}$; Simões, L. B. ${ }^{6}$; Bagagli, E.

1,2,3,4,5,6,7 Instituto de Biociências de Botucatu/UNESP - Microbiologia e Imunologia

Introduction and objectives: The dimorphic fungus, Paracoccidioides brasiliensis, is the etiological agent of paracoccidioidomycosis (PCM), a very important systemic mycosis in Latin America. The saprobic habitat of P. brasiliensis remains unknown, although some ecological studies point to soil as its possible habitat. The discovery that the nine-banded armadillo Dasypus novemcinctus is naturally infected by this pathogen represented an important step in the search for the natural habitat of the fungus. Previous studies carried out in Botucatu, an area hyperendemic for PCM, showed a high incidence of infected patients in clay soil region. Due to this preferential distribution of PCM cases, ecological studies of the pathogen in soil are necessary in order to discovery how the growth of $P$. brasiliensis is affected by the soil texture. This work aimed to evaluate the saprobe growth of $P$. brasiliensis in different textures of soil and to identify the natural habitat of this pathogen by PCR in soil from the surface and armadillo burrows. Methods and results: Soil of three textures (clay, medium and sand) was collected. Some samples of each texture were sterilized and used for culture of the yeast form of $P$. brasiliensis (three armadillo isolates: T5LN1, T9B1 and T10B1 and two human isolates: Bt84 and D01) in Petri dishes at $25^{\circ} \mathrm{C}$. The other samples were used for DNA extraction followed by specific DNA amplification by Nested PCR of rDNA regions (ITS, 5.8S). In order to study the microscopic mycelial morphology of the isolates, the fungus was also cultured in soil extract agar (SEA) and the production of conidia microscopically evaluated. The fungus growth was similar in sand and clay soil. The isolates presented some differences in growth characteristics. There was no fungus growth in soil of medium texture. The molecular detection of $P$. brasiliensis was positive in clay and sand soil collected from armadillo burrows. The microscopic observation of mycelial growth in SEA showed a large production of conidias in the isolates D01, Bt85 and T9B1. Discussion: Since growth of $P$. brasiliensis was observed in both soil textures, the high incidence of PCM in clay soil areas can be explained by the intense agricultural activities in this region, increasing the exposure to infectious aerosols. The negative growth of the pathogen in medium-texture soil can be due to some inhibitory substance present in this particular soil sample. High production of conidias from $P$. brasiliensis seeded in SEA might be explained by the presence of substances from soil that promote its sporulation and/or the low nutrient concentration of this medium, associated with inherent characteristics of each isolate, since differences were observed in both presence and quantities of conidias among the isolates. Financial support: $\mathrm{CNPq}$ PIBIC, FAPESP (grant 02/00466-5)

\subsection{8 - MOLECULAR DETECTION OF PARACOCCIDIOIDES BRASILIENSIS IN} ROAD-KILLED WILD ANIMALS FROM ENDEMIC AREAS OF PARACOCCIDIOIDOMYCOSIS: PRELIMINARY RESULTS

Richini, V. B. ${ }^{1}$; Griese, J. ${ }^{2}$; Bosco, S. M. G. ${ }^{3}$; Theodoro, R. C. ${ }^{4}$; Macoris, S. A. G. ${ }^{5}$; Silva, R. J. ${ }^{6}$; Simões, L. B. ${ }^{7}$; Bagagli, E.

1,3,4,5,7,8Instituto de Biociências de Botucatu/UNESP - Microbiologia e Imunologia; ${ }^{2,6}$ Instituto de Biociências de Botucatu/UNESP - Parasitologia

Introduction and Objectives: The ecology of Paracoccidioides brasiliensis has been poorly understood to the present. The nine-banded armadillo (Dasypus novemcinctus) plays an important role in the presence of $P$. brasiliensis in some areas endemic for paracoccidioidomycosis (PCM). PCM has been little studied in wild and/or domestic animals, which may represent an important indicator of the presence of the pathogen in nature. Molecular epidemiology has been widely used in the search for pathogens. The use of primers derived from rDNA has proved to be sensitive for pathogen detection, since these genes are present in a high copy number. The combined use of specific primers derived from this genome region with Nested-PCR may increase the molecular detection of P.brasiliensis. The present work aimed to detect possible new hosts for P.brasiliensis infection, using molecular tools. Methods and Results: Road-killed animals proceeding from Botucatu and Campinas, São Paulo State endemic region were evaluated molecularly for the presence $P$. brasiliensis in tissues. The animals and samples evaluated were: lung, spleen, liver, kidney, mesenteric lymph node, heart and adrenal gland of one of each road-killed Dasyproctidae (agouti), Erethizontidae (hedgehog) and Procyonidae (raccoon). The DNA was obtained by grinding with mortar and pestle the frozen sample with liquid nitrogen. The first round of PCR was carried out using panfungal primers ITS4/ITS5 and a specific P. brasiliensis Nested-PCR reaction using PbITSE/PbITSR primers. The amplicons were visualized by $1 \%$ agarose gel electrophoresis stained with ethidium bromide. A specific amplicon of $387 \mathrm{bp}$ was identified in samples of mesenteric lymph node, spleen, lung, adrenal gland and kidney of the agouti, liver of the hedgehog and lung of the raccoon. Conclusion: The results presented here indicate that, besides armadillos, other wild animals species can be infected by P. brasiliensis in an area endemic for PCM. The road-killed wild animals have been used for surveillance of vectors of zoonotic pathogens, such as arthropod ectoparasites. Although a serious and emerging problem in terms of conservation biology, road-killed wild animals may offer new opportunities for eco-epidemiological studies of PCM. Since it is not often possible to culture the tissues of these animals, molecular techniques can be used for detecting new hosts and mapping "hot spots" areas of PCM. Financial support: FAPESP (Grant number 02/04665)

\subsection{9 - PREVALENCES OF INFECTIONS BY PARACOCCIDIOIDES} BRASILIENSIS AND HISTOPLASMA CAPSULATUM EVALUATED BY SKIN TEST SURVEYS IN BRAZILIAN LOCALITIES: SYSTEMATIC REVIEW, META ANALYSIS AND SPATIAL APPROACHES

Mendes, R. P. ${ }^{1}$; Rodrigues, C. C. ${ }^{2}$; Simões, L. B.

${ }^{1,2}$ Faculdade de Medicina de Botucatu/UNESP - Doenças Tropicais e Diagnóstico por Imagem, ${ }^{3}$ Instituto de Biociências de Botucatu/UNESP - Microbiologia e Imunologia

Introduction and objectives: Many skin tests with histoplasmin and paracoccidioidin have been applied in Brazilian communities. However, those tests present great diversity of antigens, concentrations, sample design, intervals for readings and evaluation of the results. Thus, we conducted a systematic review aiming to uniform the methods, compare the results and assess environmental characteristics among localities. Methods and Results: The review included all published results of skin tests surveys performed in Brazil. The surveys with the same criteria were evaluated according to altitude, mean annual precipitation and temperatures of their localities. Spatial statistic was applied to assess spatial dependency among them. 
Data were statistically analyzed by the chi-square test and Pearson correlation. From 79 skin test surveys evaluated, only seven could be submitted to meta-analysis. Chi-square test has shown that the prevalence for paracoccidioidin was highest than for histoplasmin $\left(\mathrm{Cð}^{2}=47.40\right.$; $\mathrm{p}<0.0001)$. Pratânia (SP) had the highest prevalence for paracoccidioidin and Aracati (CE), for both. Teresina (PI) had the greatest frequency of negative reactions. Spatial statistic has shown that prevalence of histoplasmosis-infection does not present dependency at this scale $(\mathrm{p}>0.05)$, but paracoccidioidomycosis-infection has a global spatial pattern (Moran's I coefficient $=0.19 ; \mathrm{p}=0.02$ ). Although a small sample has been evaluated, Pearson correlation showed a trend between mean annual precipitation and prevalence of paracoccidioidomycosisinfection $(\mathrm{p}=0.09)$, when Ubatuba has not been taken in account. Prevalence of paracoccidioidomycosis-infection seems to increase in the same direction of mean annual precipitation. Conclusions: This systematic review showed the importance of a standardization of the skin test surveys for detecting the infection for both systemic mycosis. The increase in the number of studied localities and the application of the spatial statistic are necessary to better characterize the most prevalent areas for these systemic mycosis. Financial support: CAPES and FAPESP (grant 02/04489-0)

\subsection{0 - SPATIAL CLUSTERS OF PARACOCCIDIOIDOMYCOSIS IN SOUTHEASTERN BRAZIL}

Simões, L. B. ${ }^{1}$; Mendes, R. P. ${ }^{2}$; Marques, S. A. ${ }^{3}$; Pereira, P. C. M. ${ }^{4}$; Bagagli, E. ${ }^{5}$

${ }_{1,5}$ Instituto de Biociências de Botucatu/UNESP - Microbiologia e Imunologia; ${ }^{2,4}$ Faculdade de Medicina de Botucatu/UNESP - Doenças Tropicais e Diagnóstico por Imagem; ${ }^{3}$ Faculdade de Medicina de Botucatu - Dermatologia e Radioterapia

Introduction and objectives: The Region of Botucatu has been cited as an area with a high prevalence of paracoccidioidomycosis (PCM). Due to the great variation in the population size in this region, we investigated whether the high number of cases seen at the Botucatu University Hospital constitutes a statistical cluster or has occurred by chance. Methods and Results: Demographic data (annual population by gender and county) and cases by county of residence were obtained for 44 counties around Botucatu, for a 38 -year period, from 1967 to 2004. Human PCM data corresponded to the cases seen in its Services for Dermatology and Infectious Diseases. To test for the presence of disease clusters and to identify their approximate location, a spatial scan statistic was applied. Cases were assumed to be Poisson distributed, with constant risk over the space. Gender was applied as covariate. Two interations were undertaken: the first was set to include up to $50 \%$ of the total population at risk and, the second, up to $20 \%$. The calculations were performed using the software SaTScan v5.1.3. Most likely clusters were considered significant when $p<0.05$ and secondary clusters when $p<0.002$. In this period, 766 PCM-patients were evaluated, 546 of whom came from the 44 counties around Botucatu, resulting in an overall incidence rate of 2.4 annual cases per 100,000 inhabitants. The first iteration showed a geographically broad cluster with 18 counties, which reached 3.3 annual cases per 100,000 inhabitants $(p=0.0001)$, where 372 cases were observed when 272.44 were expected. The second iteration localized two spatial clusters. The most likely cluster includes Cerqueira César, with 8.7 annual cases per 100,000 inhabitants $(p=0.0001)$. The relative risk was 3.55 , with 40 cases when 11.26 were expected. The secondary cluster included Botucatu, São Manuel, Pratânia and Pardinho $(p=0.0001)$. The relative risk was 1.51 with 159 cases when 105.08 were expected $(3.7$ annual cases per 100,000 inhabitants). Conclusions: These data confirmed the Region of Botucatu as a very broad spatial cluster. This knowledge of these high incidence areas has great epidemiological importance, supporting the clinical diagnostic hypothesis and suggesting such areas as a target for investigation of the $P$. brasiliensis habitat. The clusters may represent favorable ecological conditions for the fungus and risk areas for human infection. Studies on spatial clusters using young patients with the juvenile form are ongoing. Finally, this approach could be applied in other areas endemic for PCM. Financial support: FAPESP (grants 02/04489-0 and 02/00466-5)

\subsection{1 - CANINE PARACOCCIDIOIDOMYCOSIS: CASE REPORT OF GENERALIZED LYMPHADENITIS}

Farias, M. R. ${ }^{1}$; Werner, J. ${ }^{2}$; Muro, M. ${ }^{3}$; Marques, S. A. ${ }^{4}$; Marques, M. E. ${ }^{5}$; Franco, M. ${ }^{6}$; Ribeiro, M. G. ${ }^{7}$; Custodio, C. C. ${ }^{8}$; Condas, L. A. Z. ${ }^{9}$; Bosco, S. M. G. ${ }^{10}$; Bagagli, E. ${ }^{11}$

${ }_{1,8,9}$ Pontifícia Universidade Católica do Paraná - Clínica Veterinária; ${ }^{2}$ Werner e Werner Laboratório - Curitiba/PR; ${ }^{3}$ Universidade Federal do Paraná - Hospital das Clínicas; ${ }^{4}$ Faculdade de Medicina de Botucatu - Dermatologia e Radioterapia; ${ }^{5}$ Faculdade de Medicina de Botucatu - Patologia; ${ }^{6}$ Universidade Federal de São Paulo - Patologia; ${ }^{7}$ Faculdade de Medicina Veterinária e Zootecnial UNESP Botucatu - Higiene Veterinária e Saúde Pública; ${ }^{10,11}$ Instituto de Biociências de Botucatu/ UNESP - Microbiologia e Imunologia

Introduction and Objectives: Paracoccidioidomycosis (PCM) is a subacute to chronic severe granulomatous disease, caused by Paracoccidioides brasiliensis, a dimorphic fungus endemic in Latin America. Although serological studies have been carried out in dogs, PCM disease was recently reported in a dog based on the histopathological and immunohistochemical findings and molecular detection of gp43 gene (Med Mycol. 42:379$383,2004)$. The aim of this study is to report a novel case of natural PCM in a dog, confirmed by histopathology, culture and DNA sequencing. Methods and Results: An intact female, Doberman, six years of age, was presented for veterinary assistance with history of emaciation and generalized lymphadenomegaly. A biopsy of popliteal lymph node was taken for histology, immunohistochemistry and culture. Physical examination revealed generalized lymphadenomegaly and splenomegaly. Hematological tests showed leukocytosis with neutrophilia and hyperproteinemia. Histopathological and immunohistochemical examination revealed a severe granulomatous lymphadenitis associated with numerous single or multiple budding yeasts which reacted with antibody directed against gp 43 of fungal cells. Both macro and micro aspects of culture were characteristic of $P$. brasiliensis. The fungal identification was also confirmed by gp 43 gene and ITS/5.8S rDNA sequencing. Treatment with itraconazole $(10 \mathrm{mg} / \mathrm{kg} / \mathrm{SID} / \mathrm{PO})$ was prescribed, leading to regression of the symptoms Despite the treatment, the agent still has been detected by culture and histopathology, and the present case is on medication for 10 months. Conclusion: This is the second report of canine PCM and, although it is considered rare in dogs, due to non-specific symptoms, it is believed that the incidence has been underestimated. Similarly to first case report, this occurred in a female adult Doberman, with signs of mycotic lymphadenitis. This animal presents some interesting epidemiological data. It lives in an urban area, has access to green areas and shares the space with another eight asymptomatic Doberman dogs. It is a female reproducer, which was born in Argentina and has moved to Curitiba as a puppy and has traveled for exposure and reproduction to São Paulo, Rio de Janeiro, Paraná and Santa Catarina counties. So, the source of infection, as well as the route, is difficult to determine. Despite the good response to treatment, the infection persisted and became chronic, as observed in humans, and will require long-term treatment. Curitiba is not considered an important PCM-endemic area; it has a subtropical climate with hot summers and dry humid winters, climatic conditions that favor $P$. brasiliensis growth. The identification of canine PCM should alert physicians to the necessity of investigating the epidemiological factors and occurrence of the disease in humans. 